\title{
A statistical method to identify recombination in bacterial genomes based on SNP incompatibility
}

\author{
Yi-Pin Lai and Thomas R. loerger ${ }^{*}$ (D)
}

\begin{abstract}
Background: Phylogeny estimation for bacteria is likely to reflect their true evolutionary histories only if they are highly clonal. However, recombination events could occur during evolution for some species. The reconstruction of phylogenetic trees from an alignment without considering recombination could be misleading, since the relationships among strains in some parts of the genome might be different than in others. Using a single, global tree can create the appearance of homoplasy in recombined regions. Hence, the identification of recombination breakpoints is essential to better understand the evolutionary relationships of isolates among a bacterial population.

Results: Previously, we have developed a method (called ACR) to detect potential breakpoints in an alignment by evaluating compatibility of polymorphic sites in a sliding window. To assess the statistical significance of candidate breakpoints, we propose an extension of the algorithm (ptACR) that applies a permutation test to generate a null distribution for comparing the average local compatibility. The performance of ptACR is evaluated on both simulated and empirical datasets. ptACR is shown to have similar sensitivity (true positive rate) but a lower false positive rate and higher F1 score compared to basic ACR. When used to analyze a collection of clinical isolates of Staphylococcus aureus, ptACR finds clear evidence of recombination events in this bacterial pathogen, and is able to identify statistically significant boundaries of chromosomal regions with distinct phylogenies.
\end{abstract}

Conclusions: PtACR is an accurate and efficient method for identifying genomic regions affected by recombination in bacterial genomes.

Keywords: Recombination, Compatibility, Bacteria, Evolution, Phylogenetics

\section{Background}

Recombination is an important force of evolution in prokaryotes that results in genetic exchange, usually involving transformation, transduction and conjugation [1]. In bacterial populations, when some strains acquire genetic changes from other strains, it can produce the appearance of homoplasy (where the same change at a site appears to have occurred multiple times independently, in separate branches). In a multiple sequence alignment, the polymorphic sites may have different phylogenetic relationships compared with other sites, i.e., phylogenetic incongruence [2,3]. Studies have explored the effect of recombination in phylogeny estimation and indicated that

*Correspondence: ioerger@cs.tamu.edu

Department of Computer Science \& Engineering, Texas A\&M University, College Station,TX 77843, USA

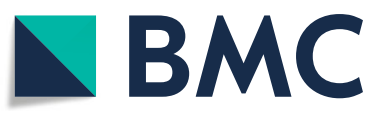

(c) The Author(s). 2018 Open Access This article is distributed under the terms of the Creative Commons Attribution 4.0 International License (http://creativecommons.org/licenses/by/4.0/), which permits unrestricted use, distribution, and reproduction in any medium, provided you give appropriate credit to the original author(s) and the source, provide a link to the Creative Commons license, and indicate if changes were made. The Creative Commons Public Domain Dedication waiver (http://creativecommons.org/publicdomain/zero/1.0/) applies to the data made available in this article, unless otherwise stated. the impact depends on the extent of recombinant events and the relatedness of taxa $[1,4,5]$. The true evolutionary history of a set of taxa may not be reflected if recombination events occurred during evolution yet are ignored. Growing evidence indicates that recombination has occurred in the evolution of many pathogenic bacterial species, including Mycobacterium avium [6], Mycobacterium intracellulare [7], Neisseria meningitidis [8, 9], Salmonella enterica [10], Staphylococcus aureus [11-13], Streptococcus pneumoniae [14] and Streptococcus pyogenes [15]. Hence, it is essential to identify recombination regions among bacterial isolates before inferring a phylogeny, to better understand their evolutionary histories.

Over the last four decades, many methods have been proposed to detect the presence of recombination in 
bacterial genomes, applying concepts of maximum likelihood, phylogenetic incongruence, substitution patterns, distance-based approach, or character compatibility [16-22]. Commonly used methods to identify recombination breakpoints include ClonalFrameML [22], RDP [18] and GARD [19]. All are phylogenetic-based programs. ClonalFrameML utilizes a maximum-likelihood tree to reconstruct ancestral states of internal nodes. It then applies a hidden Markov model (ClonalFrame) to infer the recombination parameters and recombination locations of each branch of the tree using an Expectation-Maximization (EM) algorithm [22]. RDP characterizes homoplasy signals using pairwise scanning of the alignment, with the integration of several non-parametric recombination detection methods [18]. GARD applies Akaike's Information Criterion with a genetic algorithm to search the recombinant locations heuristically [19]. Compatibilitybased methods are considered to be more efficient than phylogenetic-based methods to identify recombination, since they do not require the reconstruction of phylogenetic trees [16]. The Reticulate program uses compatibility matrices to calculate a neighbor similarity score (NSS), and clusters compatible sites by randomly shuffling the matrices [17]. Bruen et al. define the pairwise homoplasy index (PHI) in terms of a pairwise incompatibility score of each site and its downstream sites in a global alignment, and then they obtain a $p$-value by computing the cumulative probability under a normal distribution generated from expected mean and variance of the PHI statistic [20]. Both programs are compatibility-based methods and able to detect recombination and report informative sites, but they do not report breakpoints.

(paragraph on compatibility integrated into Methods...)

In our previous work, an average compatibility ratio (ACR) method was introduced to identify the potential recombination breakpoints in a bacterial genome by analyzing the pattern of SNPs among a collection of isolates using a sliding window [23]. The ACR method detects the presence or absence of recombination by calculating an overall compatibility score among pairs of sites. Next, ACR will scan the entire alignment with a sliding window of fixed size to identify regions where the local compatibility among pairs of sites in the region decreases and reaches a local minimum. However, the local minima may include false positives. In this paper, we propose the use of a permutation test on the positions of local minima to assess the statistical significance of potential breakpoints in the genome. We also extend the ACR method to test the compatibility of multi-state characters by applying an efficient algorithm based on Buneman's theorem [24]. The performance of ptACR is evaluated on simulated datasets with varying mutation rates and rate heterogeneity among sites. The sequences are simulated by evolving along distinct trees with changes in topology, where a group of taxa have been moved from one branch to another randomly. The simulation results show that the integration of the permutation test has lower false positive rate than basic ACR method. Yet both methods have a similar level of sensitivity for the detection of recombination breakpoints. We use ptACR to identify genomic regions of recombination in clinical isolates of Staphylococcus aureus.

\section{Methods}

\section{Characters and compatibility}

The concept of compatibility was initially described by LeQuesne in 1969 for binary-state characters [25]. For a multiple DNA sequence alignment, a character is defined as a set of states (nucleotides) for all taxa at a given site. A binary character is a polymorphic site with 2 nucleotides. Two binary-state characters are compatible if a single phylogenetic topology is enough to explain both characters:

Definition 1 Pairwise compatibility for binary characters: Two sites of binary characters are compatible if and only if there exists a tree for which each site can be explained by one change.

For a pair of binary characters at two sites, the four gamete test is a quick way in polynomial time to determine their compatibility [26]. It converts the state of taxa at each site to 0 and 1 , and concatenates the states at two sites for a given taxon as one of the following combinations: $\{00,01,10,11\}$. If at most three combinations exist, then the two sites are compatible. For a set of binary characters in an alignment, there exists a perfect phylogeny if all characters are jointly compatible. For a set of 3 or more binary-state sites in a region of genome, if all pairs of sites are pairwise compatible, then they are jointly compatible, i.e. a tree exists that can explain all sites.

More generally, in a whole-genome alignment of multiple taxa, some sites can also have multiple states, e.g., 3 or 4 nucleotides.

Definition 2 Pairwise compatibility for multi-state characters: Two sites of multi-state characters are compatible if and only if there exists a tree for which each site can be explained by number of change that equals to number of distinct states minus one (the minimum number of changes required for a site with $n$ nucleotides is $n-1$ ).

To determine the compatibility of a pair of multi-state characters (two sites at a time), the problem can be reduced to triangulating colored graphs problem [27] and then solved in polynomial time [24]. Two characters are firstly converted to a partition intersection graph by the following steps. For each character, the taxa of the same state are denoted as a vertex. An edge between two vertices is added if the vertices contain the same taxon/taxa 
to form the partition intersection graph. Next, if their derived partition intersection graph is acyclic, then they are determined to be compatible [24]. The method to determine the compatibility of two characters is illustrated in Algorithm 1. For multi-state characters, pairwise compatibility does not guarantee setwise compatibility.The question of determining whether a set of $n>2$ multi-state sites is compatible is reducible to the problem of finding the maximum clique, which is NP-complete [24].

$\overline{\text { Algorithm } 1 \text { Pairwise compatibility of two multi-state }}$ characters

Require: Characters $\chi_{p}$ and $\chi_{q}$ at the site $p$ and site $q$

Ensure: True if they are jointly compatible and False if they are incompatible;

function CHARCOMPAT $\left(\chi_{p}, \chi_{q}\right)$

Collect the sets of taxon/taxa of the same state (nucleotide), where the number of unique states are denoted as $r_{1}$ and $r_{2}$ :

$$
\begin{aligned}
& \chi_{p}^{\prime} \leftarrow\left\{x_{i}\right\}, i=1, \ldots, r_{1} \\
& \chi_{q}^{\prime} \leftarrow\left\{y_{j}\right\}, j=1, \ldots, r_{2}
\end{aligned}
$$

Initialize an undirected graph $G$ by the adjacency list Add sets in $\chi_{p}^{\prime}$ and $\chi_{q}^{\prime}$ as nodes to $G$

Add an edge between node $u$ and node $v$ by $G(u, v)$ to update the graph $G$ :

$$
\begin{aligned}
& \text { for all } x_{i} \text { in } \chi_{p}^{\prime} \text { do } \\
& \text { for all } y_{j} \text { in } \chi_{q}^{\prime} \text { do } \\
& \text { if } x_{i} \cap y_{j} \neq \emptyset \text { then } \\
& G \leftarrow G\left(x_{i}, y_{j}\right) \\
& \text { end if } \\
& \text { end for } \\
& \text { end for }
\end{aligned}
$$

Check for cycles in $G$ by depth first search (DFS)

return True if there is no cycle in G, False otherwise end function
Given a multiple sequence alignment of $n$ taxa and $m$ informative sites, at each informative site $i$, ACR calculates a pairwise compatibility score between all pairs of informative sites within a sliding window of size $2 w$ centered on the $i^{\text {th }}$ SNP (from $i-w$ to $i+w$ ). The pairwise compatibility score is 1 if characters $\chi_{p}$ and $\chi_{q}$ are compatible; otherwise, the score is 0 (Eq. 1). Next, it averages the scores of all pairs of sites within the region to obtain the average compatibility ratio, $\sigma_{i_{w}}$, for the region (Eq. 2).

Compat $P W_{p q}=\left\{\begin{array}{l}1, \text { if characters } \chi_{p} \text { and } \chi_{q} \text { are compatible } \\ 0, \text { otherwise }\end{array}\right.$

$$
\sigma_{i_{w}}=\frac{1}{\left(\begin{array}{c}
2 w+1 \\
2
\end{array}\right)} \sum_{p=i-w}^{i+w-1} \sum_{q=p+1}^{i+w} \operatorname{Compat} W_{p q}
$$

The lower the value of the average compatibility ratio $\left(\sigma_{i_{w}}\right)$, the less jointly compatible the sites in a window are. Hence, a site of local minimum means that sites in the region are least compatible locally, suggesting phylogenetic incongruence between the upstream and downstream regions. Sites with local minima of average compatibility ratio are regarded as potential breakpoints. An example of applying ACR on a recombined alignment of 5200 sites using the window size of 200 is demonstrated in Fig. 1.

To assess the statistical significances of potential breakpoints, we apply a permutation test. The test statistic, $s_{i_{w}}$, for a potential breakpoint at the site $i$ is defined as the summation of all compatibility scores of pairs composed of a site from the upstream region $[i-w, i-1]$ with the other site from the downstream region $[i+1, i+w]$ (Eq. 3).

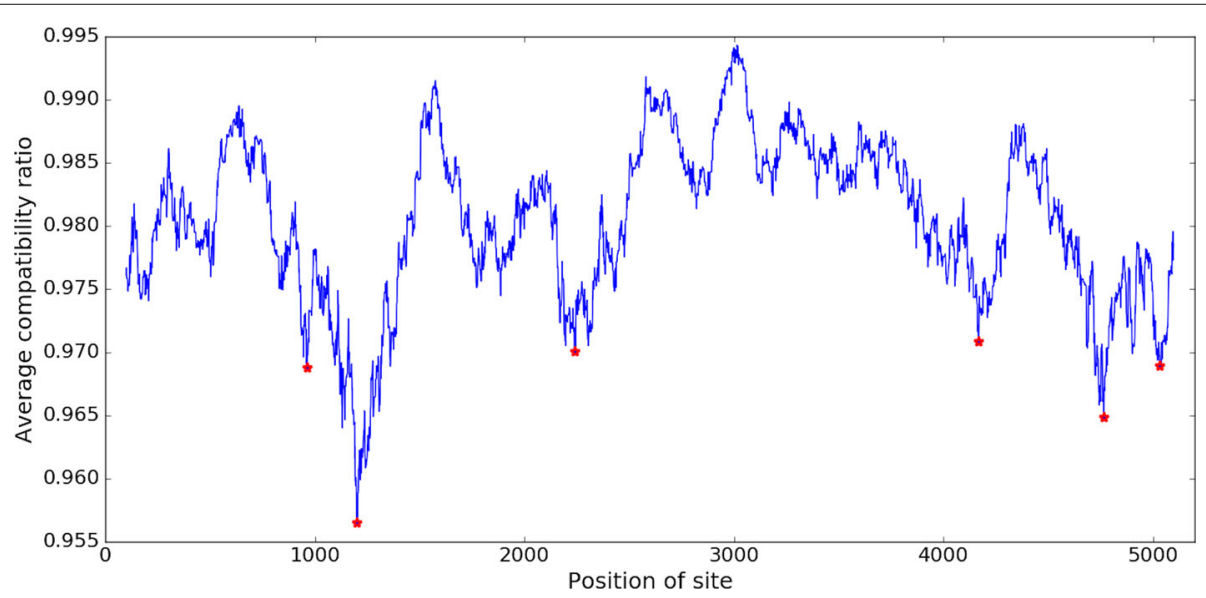

Fig. 1 Example of applying ACR on an alignment of several recombined regions using the window size of 200. Among 5200 sites, six sites are identified as the potential breakpoints and labeled in red 


$$
s_{i_{w}}=\sum_{p=i-w}^{i-1} \sum_{q=i+1}^{i+w} \text { CompatP } W_{p q}
$$

This statistic is compared to a null distribution generated by permuting the sites in the window. The null hypothesis is that the level of compatibility between the sites in the window is independent of the sequential order of the sites, i.e. whether sites are compared from upstream or downstream of site $i$ does not matter. The alternative hypothesis is that the order of the sites in the local sequences is crucial and does not happen by chance. So the sites within the region are randomly shuffled multiple times (default: 10,000) to produce the sampling distribution of values $s_{i_{w}}$ obtained under the null hypothesis. Let the distribution of values from random permutations on sites in the window be denoted by $D_{s}$. The significance of observed value $s_{i_{w}}$ is determined by computing the proportion of times that the permuted statistics in $D_{s}$ are less than or equal to the observed value to get the empirical $p$-value (Eq. 4).

$$
p=P\left(x \leq s_{i_{w}} \text { for } x \in D_{s}\right)
$$

If the $p$-value is lower than a given threshold (default: 0.05), then it rejects the null hypothesis of no recombination, hence ptACR will report the site as a probable/significant breakpoint. To correct the $p$ value threshold due to multiple comparison, we use the Bonferroni correction and set the adjusted $p$-value cutoff to $0.05 / n$, where $n$ is the number of local minima identified by ACR, to limit the false discovery rate to at most
$5 \%$. An example of a statistic determined as significant in the histogram of a null distribution is illustrated in Fig. 2. To make the permutation test more efficient, we convert all characters in nucleotides of the alignment to patterns in numbers and make character patterns as a unique set. Then we record pairwise compatibility information among all pairwise patterns in the set in a hash table. Hence, the compatibility information of any two shuffled sites can be looked up in the hash table in constant time.

\section{Estimation of phylogenies and homoplasy}

Given a sorted list of candidate breakpoints, local phylogenetic trees of each region between two adjacent breakpoints is constructed by the maximum parsimony method using the function of dnapars in PHYLIP 3.66 [28]. To estimate the level of homoplasy for each region, the homoplasy ratio and excess changes are calculated by applying the Sankoff Algorithm [29] on each local tree. The homoplasy ratio, which is also called the ratio of changes per site, is defined as the summation of actual state changes (Sankoff score) divided by the summation of minimum number of changes (number of nucleotides at each site minus one). The number of excess changes for a site is defined as the difference between the number of actual changes and minimum number of changes. For a given region, the homoplasy ratio of 1.0 means all sites are congruent (homoplasy-free); a homoplasy ratio $>1.0$ means some sites are homoplasic, requiring excess changes in the maximum-parsimony tree.

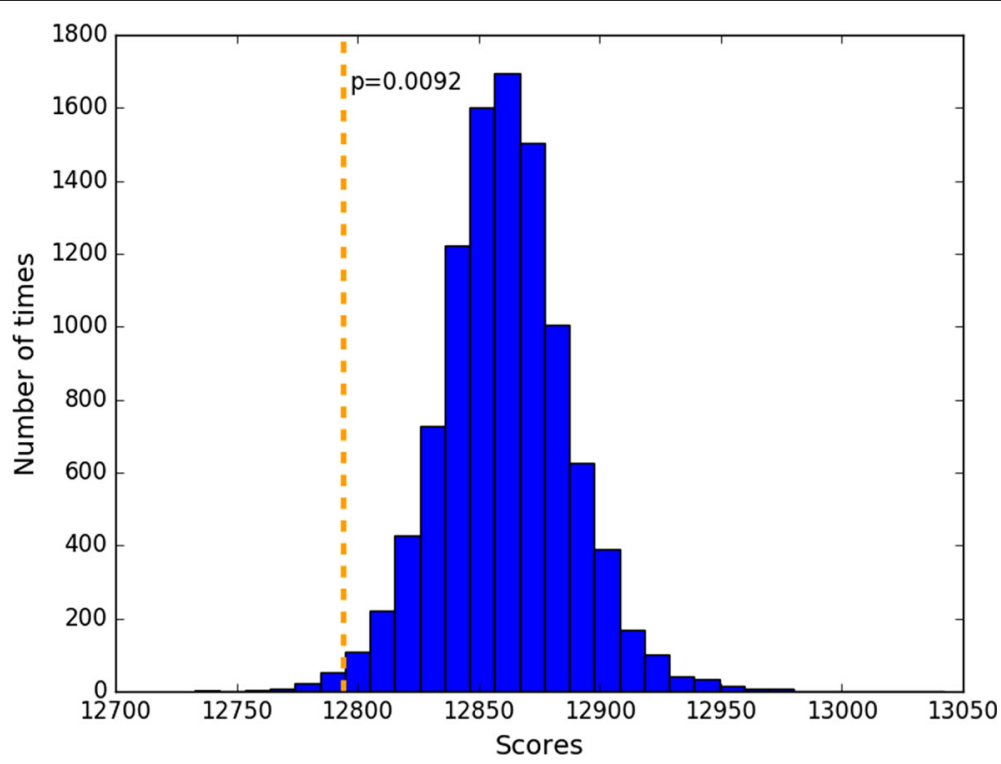

Fig. 2 Example of the assessment of statistical significance for a compatibility score in the histogram of a null distribution ( $\mathrm{N}=10 \mathrm{k}$ ). Observed compatibility score at the site $i$ was 12800 , among pairs selected upstream and downstream sites. Distribution shows scores from randomly selected pairs in window of $[i-w, i+w]$. The $p$-value in this case is 0.0092 (at the tail) 


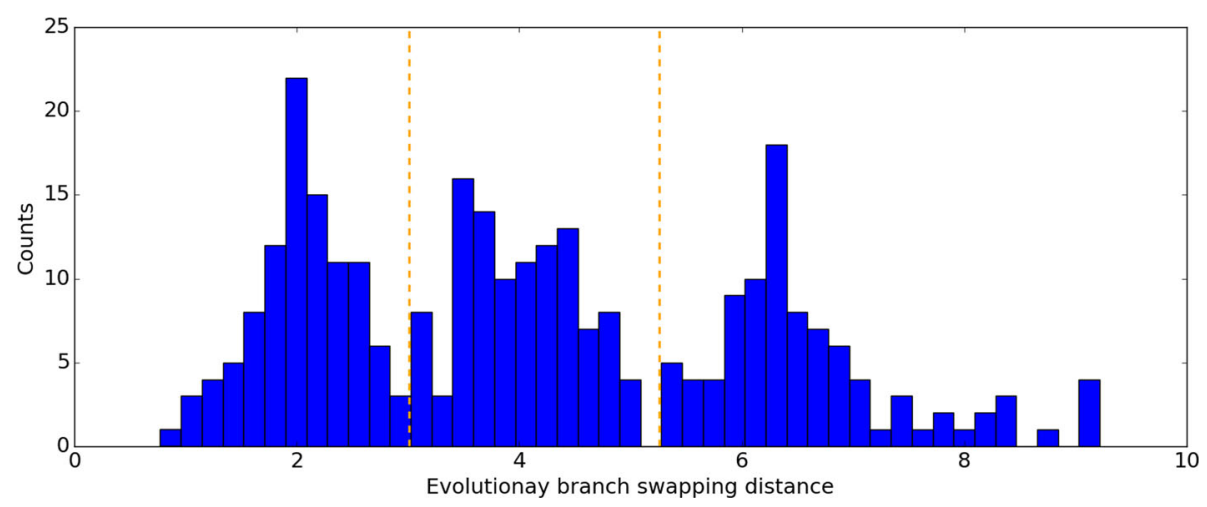

Fig. 3 Histogram of evolutionary branch swapping distance between the original tree and 300 alternative trees generated using HGT-Gen

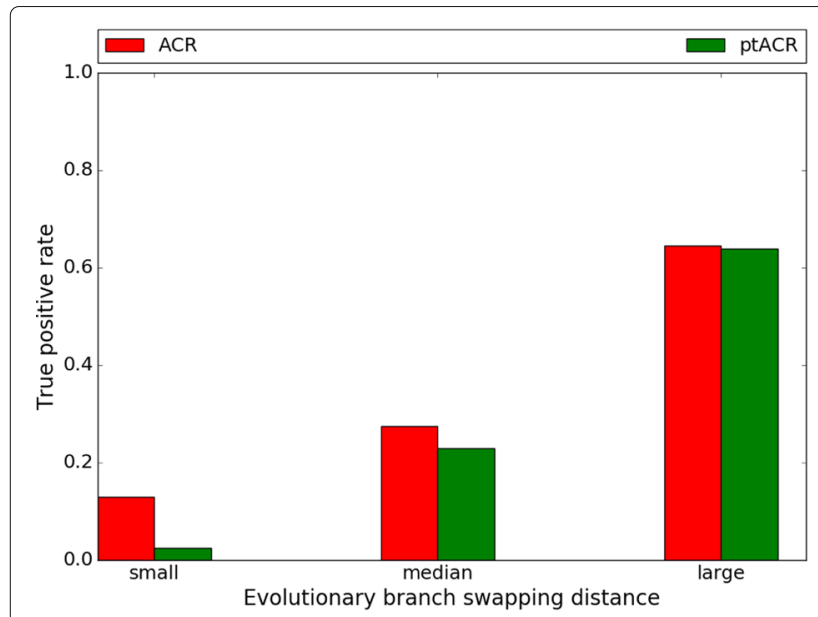

(a)

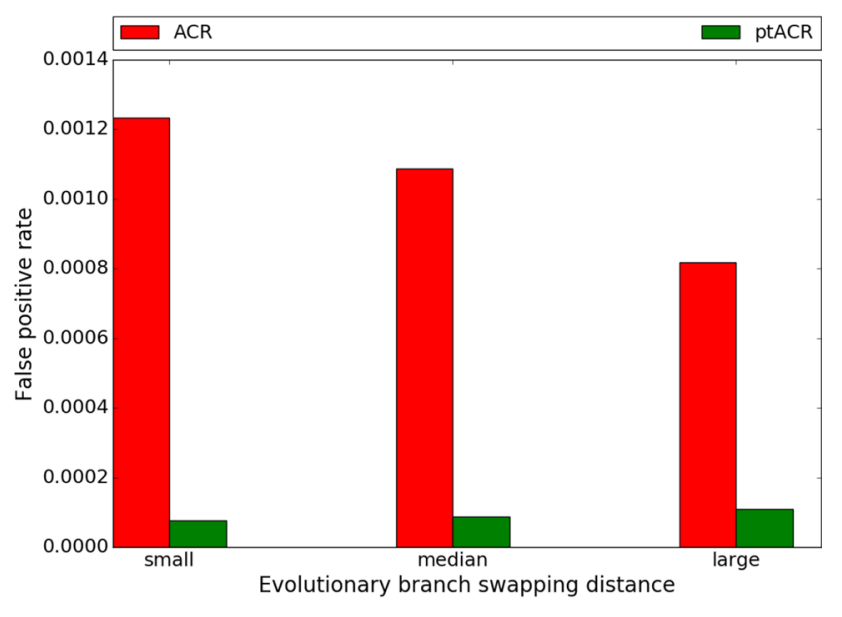

(b)

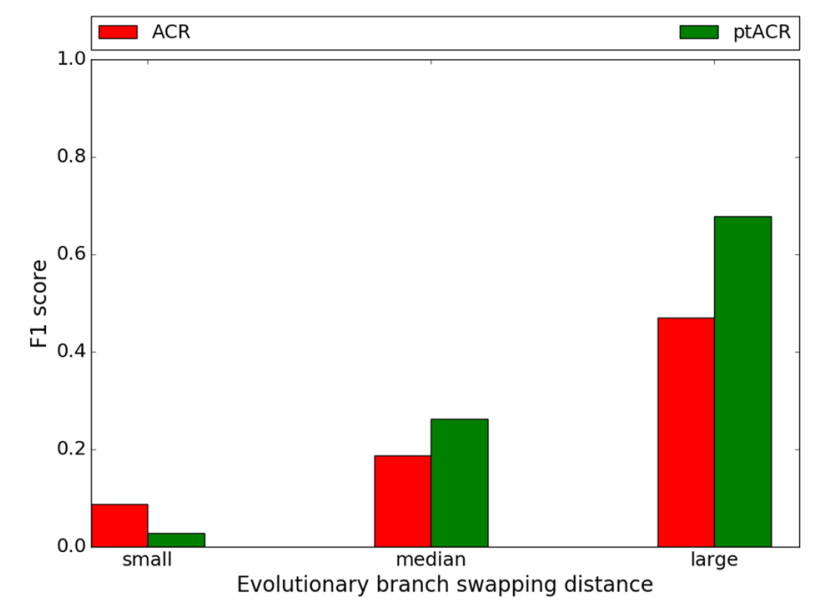

(c)

Fig. 4 True positive rate (a), false positive rate (b) and F1 score (c) of 3 scenarios of increasing evolutionary branch swapping distance (no heterogeneity) 


\section{Performance on simulated datasets}

To evaluate the performance of ptACR, we generated simulated sequence data with known recombinations by random branch swaps. Our goal was to evaluate the sensitivity and specificity of detecting known breakpoints, and how this depends on mutation rate and differences in topology. To simulate sequences with predetermined recombination events, a bifurcating tree with 10 taxa is generated by GenPhyloData [30] under a birth-death process with a birth rate of 0.2 and a death rate of 0.1 . Next, 300 alternative trees with recombination between a random pair of donor and acceptor branches based on the original tree are obtained using HGT-Gen [31]. Then, Seq-Gen 1.3.4 [32] is applied to generate aligned sequences of 1000 sites evolved along each tree. Parameters for substitution rate and heterogeneity are varied in the experiment, as described below. The sequences are simulated under the Hasegawa-Kishino-Yano model (HKY85) [33] with nucleotide frequencies A:0.2, G:0.3, C:0.3, T:0.2 and 2-to-1 ratio of transitions to transversions. Lastly, we concatenate sequences for the original tree, one of the modified trees, and the original tree again to obtain a simulated alignment with 3000 total sites that has recombination breakpoints around coordinates 1000 and 2000 and a distinct phylogeny in the middle.

The true positive rate (sensitivity), false positive rate (1-specificity), and F1 score for the ptACR method are defined as follows. For an alignment with a predetermined recombination region, the inferred breakpoint that is located within $50 \mathrm{bp}$ of an actual breakpoint (ground truth) is counted as true positive (TP), and one that is identified by our method but not within this range is denoted as false positive (FP). Failure to detect a known breakpoint at any site within $50 \mathrm{bp}$ is counted as false negative (FN). The true and false positive rates are defined by dividing by the total number of true breakpoints, and the total number of negative sites outside the breakpoint windows, respectively, $\frac{T P}{T P+F N}$ and $\frac{F P}{F P+T N}$. The precision is defined as the number of accurately inferred breakpoints to the number of identified breakpoints, $\frac{T P}{T P+F P}$. The F1 score, which is the harmonic mean of sensitivity and precision, is $\frac{T P}{2 T P+F P+F N}$; higher $\mathrm{F} 1$ is better. For each scenario, we average the statistics over all the replicates.

\section{Effect of evolutionary distance}

Because recombination events among deeper branches should involve strains with more differences and make incompatibility easier to detect, we expect that sensitivity and specificity will vary as a function of the magnitude of the changes in the simulated trees. To quantify this, we defined an metric called evolutionary branch swapping distance (EBSD) to divide the alternative trees into 3 groups: small, medium, and large evolutionary changes. While there are several generalized methods for comparing topologies of arbitrary labeled trees (sharing the same taxa) [34-36], assuming that the change between two trees involves only a single branch swap (as generated by HGT-Gen, simulating recombination), we developed a quantitative measure that reflects the magnitude of evolutionary distance involved in the change. First, we identify the group of taxa that changes position in the tree. Call this group $\mathrm{A}$, and let $\mathrm{B}$ be the complement in the tree (rest of the taxa). We define the evolutionary branch swapping distance between the two trees (T1 and T2) as the average absolute value of the difference in distances between each pair of taxa $i$ in A and $j$ in B in trees T1 and T2 (Eq. 5).

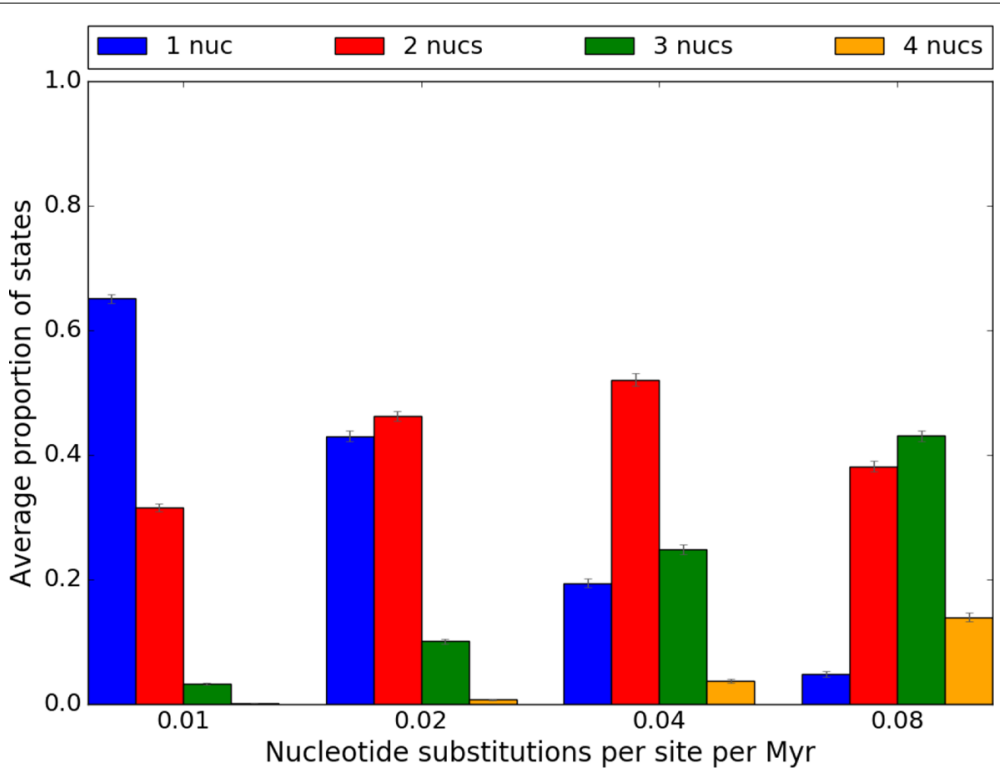

Fig. 5 Proportion of nucleotides in 4 scenarios of increasing substitution rate 
$\operatorname{EBSD}(T 1, T 2)=\frac{1}{|A| *|B|} \sum_{i \in A} \sum_{j \in B}\left|\operatorname{dist}_{T 1}(i, j)-\operatorname{dist}_{T 2}(i, j)\right|$

The distance between two taxa is defined as the sum of branch lengths on the connecting path in a tree. The distances between pairs of taxa that are both in A or both in $B$ should be unaffected by the branch swap; only pairs of strains between the two groups will exhibit changes in relative position, and hence changes in distance. If a strain (or group of strains) recombines with a nearby branch, the average change of distances among them will be small; however, if they recombine with a more remote branch of the tree, representing exchange of genetic material with a more divergent strain, then the change in relationships will be more pronounced, and the average change in relative distances among the strains will be larger. The distribution of EBSD distances between the original tree and the 300 alternative trees ranged from 0.77 to 9.22 (see histogram in Fig. 3). The alternative trees are categorized into three groups according to the tree distance with the original one, including small $(<3.0)$, medium (3.0-5.0) and large distance $(>5.0)$ groups. There are about 100 trees in each category.

The true positive rate, false positive rate and F1 score of replicates in the three groups are shown in Fig. 4. Importantly, there is a great reduction in false positives (Fig. 4b) without much loss of true positives (Fig. 4a) for ptACR on ACR. In general, a replicate in the large evolutionary branch swapping distance group has sequences simulated from a more distinct alternative topology compared to the original tree, which makes the sites in the middle of the alignment tend to exhibit more homoplasy. Thus, the boundaries of the recombination event are easier to

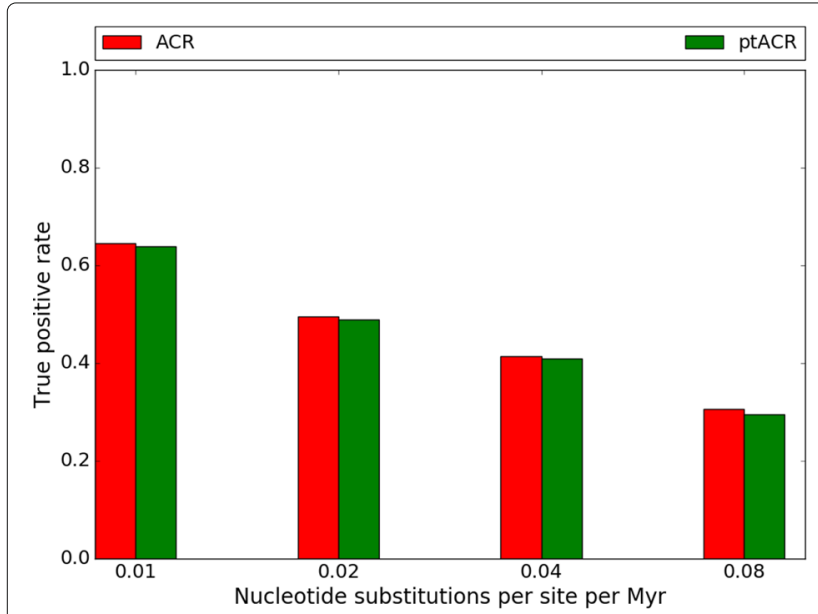

(a)

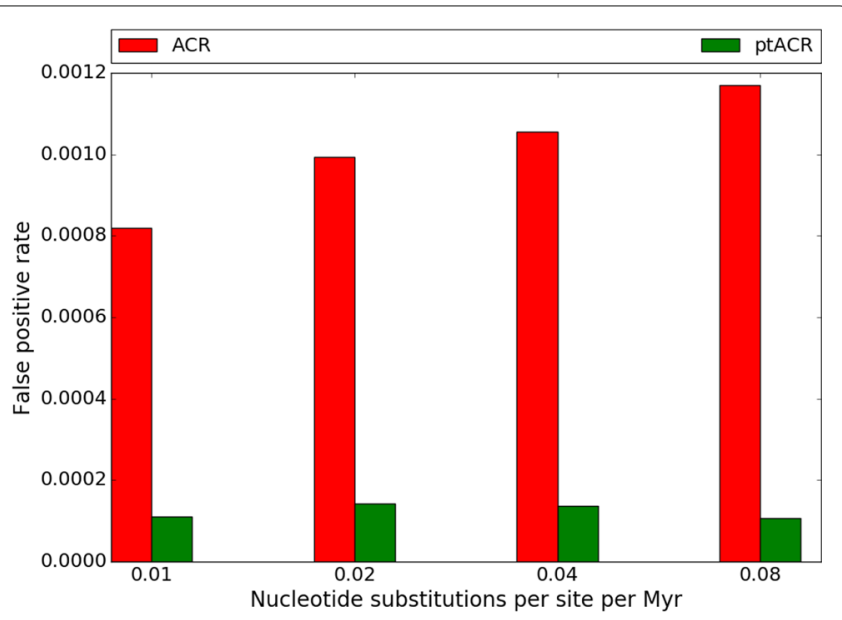

(b)

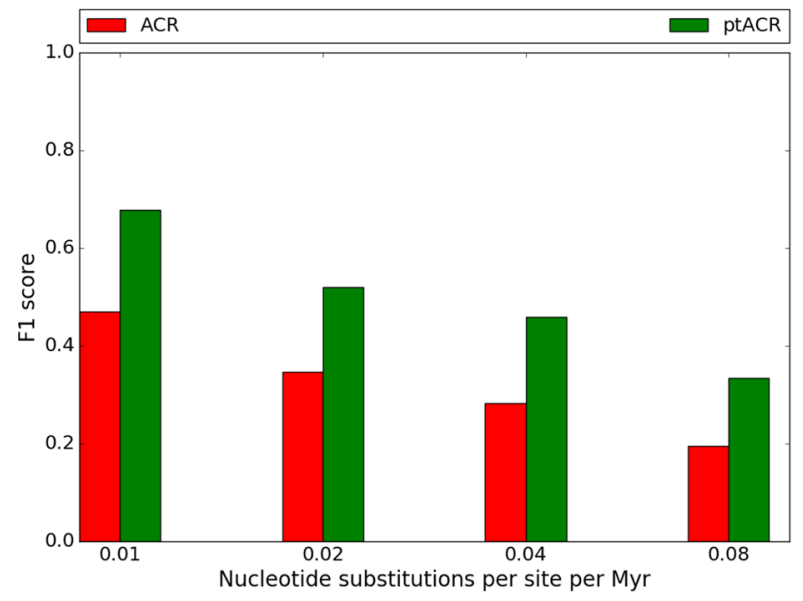

(c)

Fig. 6 True positive rate (a), false positive rate (b) and F1 score (c) of 4 scenarios of increasing substitution rate 
detect. In contrast, replicates in the small distance group have closer relatedness of taxa since the alternative tree is less different to the original tree. As evolutionary branch swapping distance decreases, both sensitivity and specificity are reduced.

\section{Effect of substitution rate and heterogeneity}

Sequences were simulated in four scenarios by setting the substitution rate parameter of Seq-Gen to 0.01, 0.02, 0.04 and 0.08 . Only recombined trees in the large evolutionary branch-swap distance group were used in this experiment, as the sensitivity of ptACR is higher. The default substitution rate heterogeneity parameter in Seq-Gen was used $(\alpha=\infty$, which means no heterogeneity). The proportion of nucleotides in each scenario is shown in Fig. 5. With low substitution rate, there are $62 \%$ monomorphic sites. As substitution rate increases, the fraction of informative sites increases. The true positive rate, false positive rate and F1 score of the four scenarios are plotted in Fig. 6. With low substitution rate, the true positive rate is high, the false positive rate is low and the F1 score is high. The ptACR approach performs better than the ACR in terms of lower false positive rate and higher F1 score.

To examine how substitution rate heterogeneity affects ptACR performance, we varied the heterogeneity $\alpha$ (shape parameter of the gamma distribution) in Seq-Gen, which influences the variability of substitution rates among individual sites. Sequences are simulated in four scenarios of heterogeneity parameter $\alpha$ ranging from $0.2,0.8,1.6$ to $\infty$ (with the fixed substitution rate of 0.01 ). The scenario where $\alpha$ is equal to $\infty$ represents sequences simulated with a uniform rate at all sites. The proportion of nucleotides in alignments in each scenario is listed in Fig. 7. With low heterogeneity $(\alpha=\infty)$, there are $37 \%$ polymorphic sites and $12 \%$ of there are multi-state characters. As heterogeneity increases, the fraction of informative sites decreases. The true positive rate, false positive rate and F1 score of four scenarios are plotted in Fig. 8. The red bars stand for the results from the previous ACR method while the green bars show the results of incorporating the permutation test (ptACR). With low heterogeneity, the true positive rate is high, the false positive rate is low and the F1 score is high. Only at the highest heterogeneity are the sensitivity and specificity reduced. Hence, ptACR accurately detects recombination breakpoints in the alignments, including multi-state characters, except in the most extreme divergent situations (where there is more background homoplasy) occurring stochastically even without recombination.

\section{Results}

We applied ptACR to analyze a collection of 30 clinical isolates of Staphylococcus aureus [12] aligned with 5 reference strains, including ST8:USA300 (NC_010079.1), SACOL (CP000046.1), EMRSA-15 (HE681097.1), N315 (BA000018.3) and ATCC 25923 (NZ_CP009361.1). Recombination has previously been observed for the species [12, 13]. The alignment of Staphylococcus aureus contains 2.87 $\mathrm{Mb}$ nucleotides where 113,936 sites are informative (polymorphic) and 3,625 sites (3.18\%) have over two nucleotides. The overall compatibility ratio over the genome is $88.34 \%$ and the homoplasy ratio

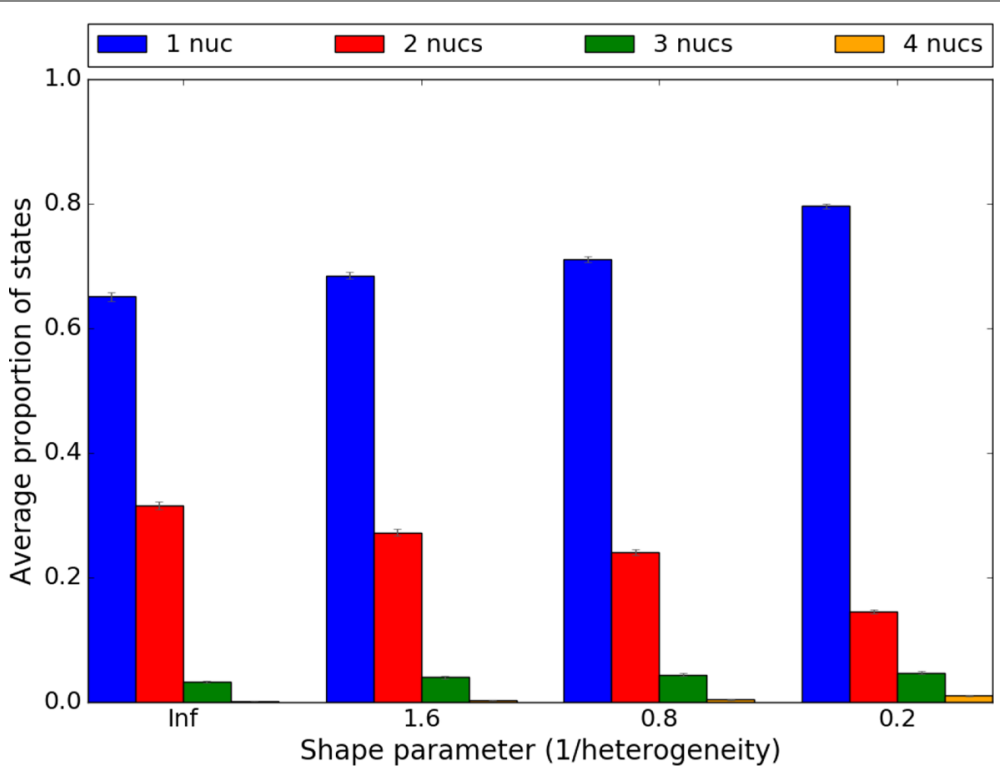

Fig. 7 Proportion of nucleotides in 4 scenarios of increasing heterogeneity 
is 1.4484, suggesting recombination occurs among the population. The global phylogenetic tree is shown in Fig. 9.

Figure 10 illustrates that 86 local minima (labeled in red) are identified by ACR as potential breakpoints using a window size of 250 informative sites, and then 65 breakpoints (labeled in green) are identified as statistically significant by ptACR with permutation test, where the Bonferroni-adjusted $p$-value threshold is 0.000581 $(0.05 / 86)$. Hence, 66 regions are obtained.

Any two adjacent regional phylogenetic trees constructed by their corresponding local alignments have distinct tree topologies, reflecting the identified boundaries are confident, since changes in phylogenetic relationships occur between each pair of adjacent regions.

The plots of the homoplasy ratio and the excess changes for each region based on the global tree and a regional tree are shown in Fig. 11. For each region, both homoplasy ratio and excess changes decrease from the global tree to the regional tree, showing that the regions identified by ptACR have different topologies from the global tree, and each local tree is able to accommodate more sites within the corresponding region. Figure 12 shows local phylogenetic trees for three consecutive regions, starting from the $37^{\text {th }}$ segment, as an example for further analysis. The recombined groups of isolates are labeled in rectangles of the same color. According to the tree topologies, the $37^{\text {th }}$ region shows that the strain ERR410042 receives a copy from an ancestor of two strains, ERR410056 and ERR410060. Yet in the $38^{\text {th }}$ region the strain ERR410042 receives a copy from an ancestor of three strains, ERR410044, ERR410046 and N315, while a parent of ERR410056 and ERR410060 receives a copy from an ancestor of ERR410038, ERR410039 and EMRSA15 . In the $39^{\text {th }}$ region the strain ERR410042 receives the copies from parents of the strain ERR410058 instead. The

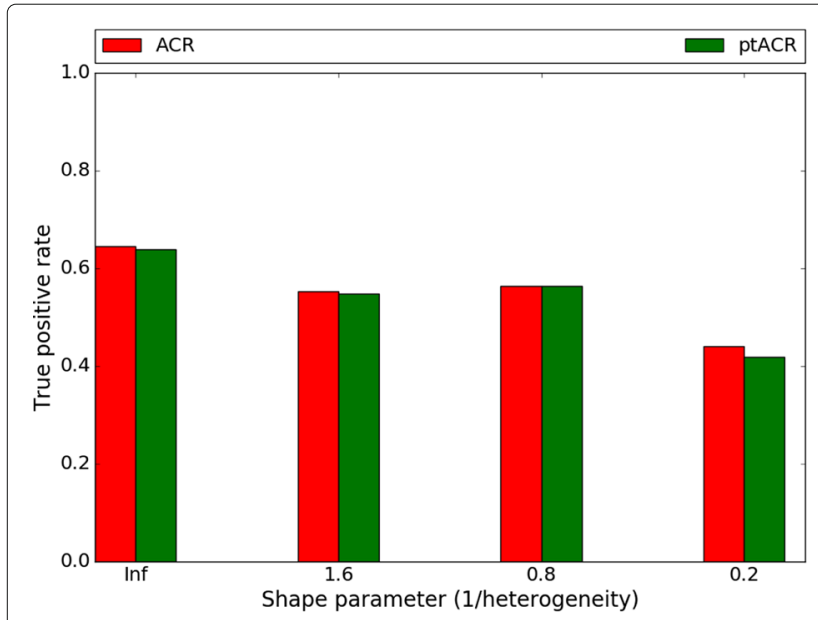

(a)

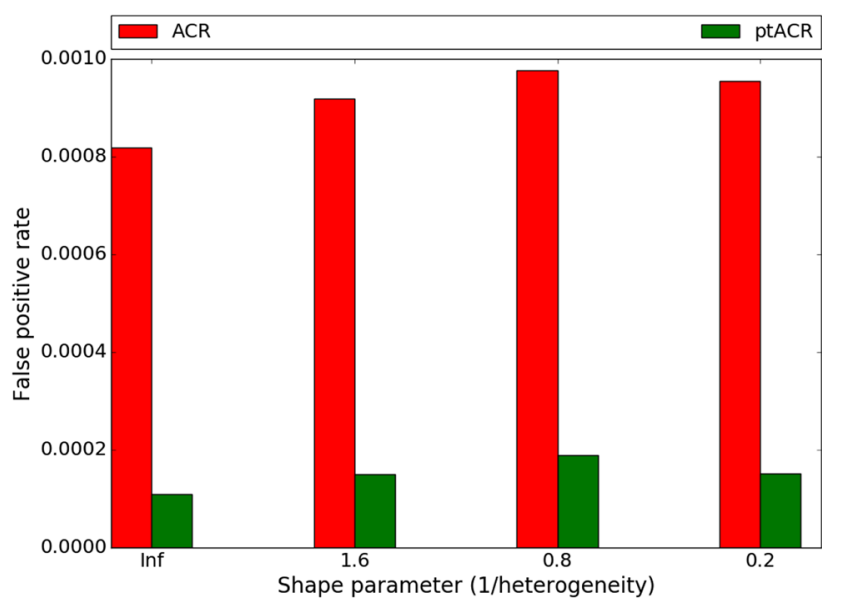

(b)

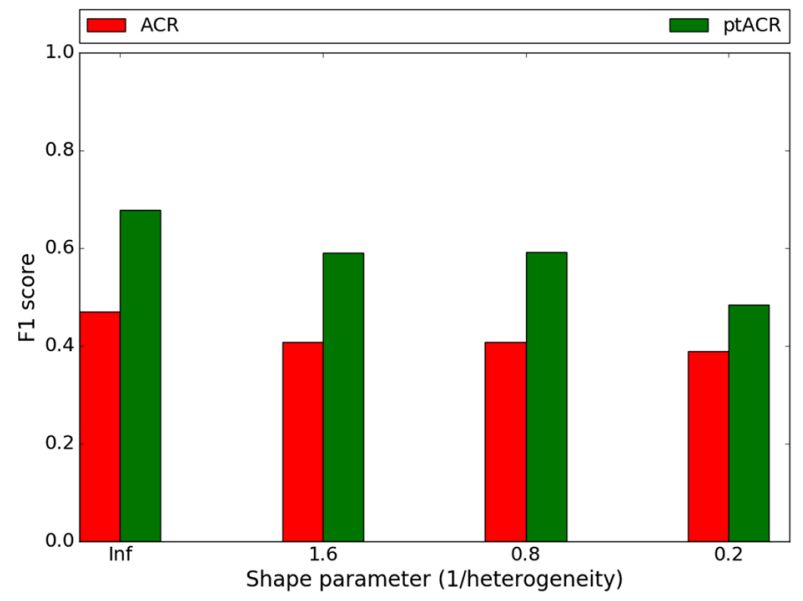

(c)

Fig. 8 True positive rate (a), false positive rate (b) and F1 score (c) of 4 scenarios of increasing heterogeneity (fixed substitution rate) 


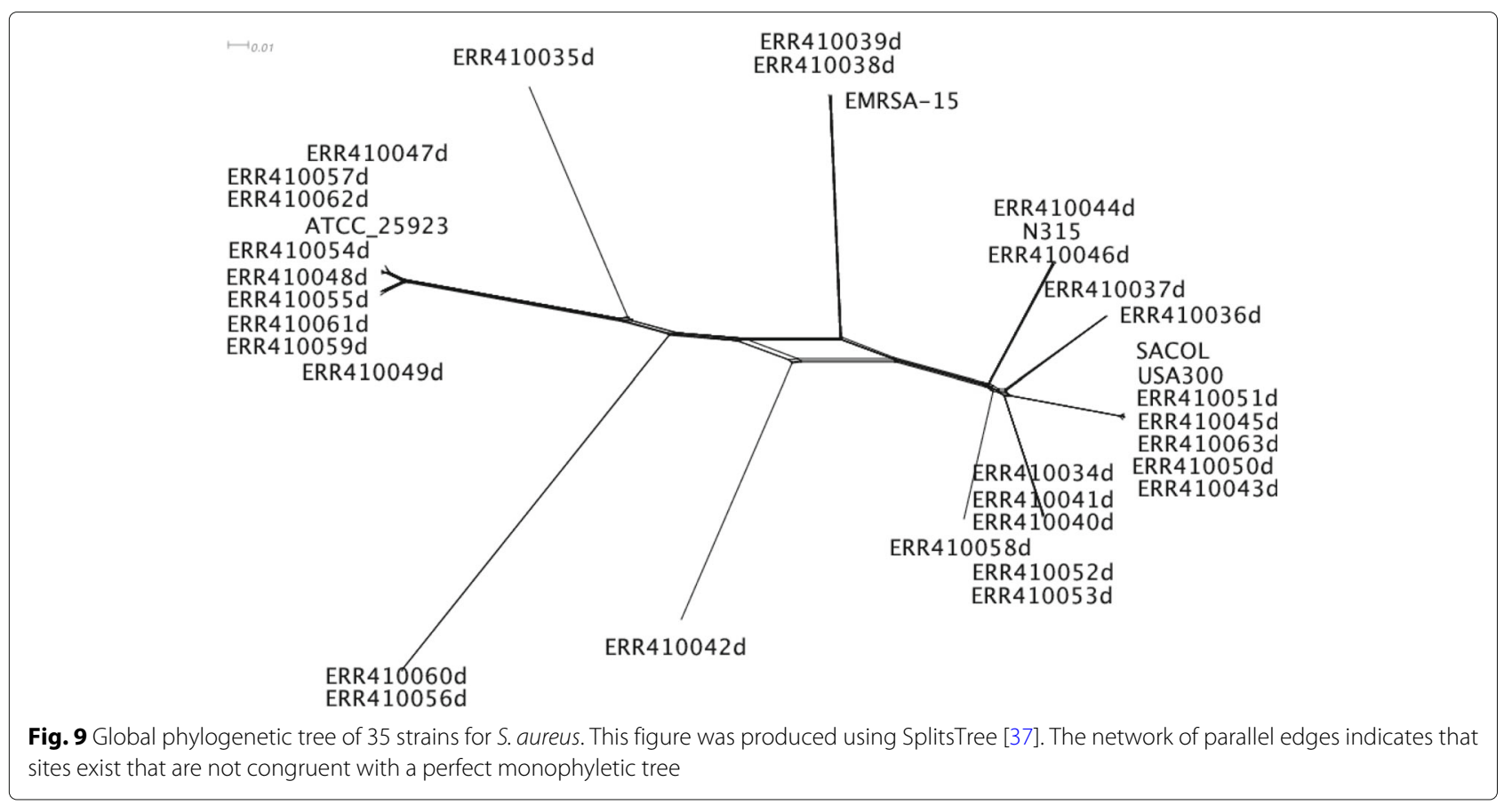

information of region size, number of informative sites (SNPs), genes, overall compatibility ratio (Compat), the excess changes based on global tree $\left(E C_{\text {global }}\right)$ and local tree $\left(E C_{\text {local }}\right)$, and the reduction ratio of excess changes (Ratio) for the three regions is listed in Table 1. The number of excess changes decreases from the global tree to the local tree, showing that the local trees significantly reduce the apparent homoplasy based on the global tree.

(paragraph on S. aureus moved to Discussion...)
To visualize the relationships among strains, a plot of the most closely related reference strain for each strain in each region is shown in Fig. 13. Strains ST8:USA300, EMRSA-15, ATCC 25923 and N315 were used as references, spanning several different lineages/strain types worldwide. For each strain, the most closely related reference strain is defined as the one that has the least differences in a region. Figure 13 shows that for several strains, the most closely related reference strain changes

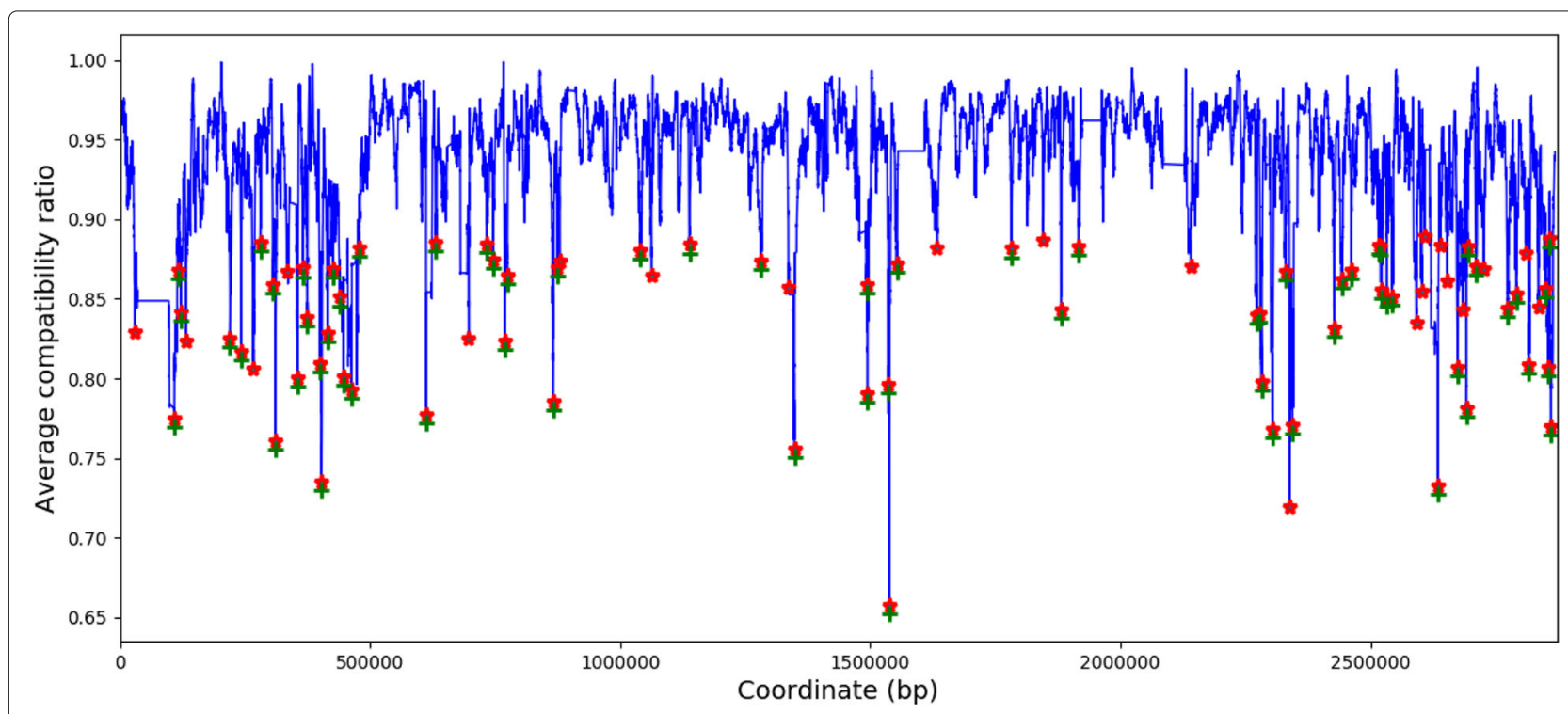

Fig. 10 Identified breakpoints using window sizes of 250 informative sites for S. aureus 
across the genome (i.e., pattern is mosaic), indicating that they are likely recombined (especially ERR410042). This is consistent with previous studies that found extensive recombination in this collection of $S$. aureus isolates $[12,13]$.

\section{Discussion}

Our evaluation of ptACR demonstrates that it is not only sensitive to the true positives but robust to the false positive signals. Experiments with simulated data show that the sensitivity of ptACR increases for recombination between more divergent strains (with higher evolutionary distance). The performance is also influenced by substitution rate and heterogeneity. Including substitution rate heterogeneity among sites is more biologically realistic since some essential genes are more conserved while other genes tolerate more genetic changes during evolution over time. The extent of substitution rate heterogeneity is inversely related to the shape parameter in the gamma distribution. As the substitution rate heterogeneity decreases, more informative sites are obtained, and the ptACR performs better in terms of true positive rates. However, in the scenarios of higher substitution rate heterogeneity, there are more false positive signals. With the assessment of statistical significance of breakpoints using the permutation test, ptACR outperforms our previous method by filtering out more false positive signals. Also, substitution rate is varied to explore the influence of selection and genetic drift during evolution. When the substitution rate is lower, the true positive rate is higher and false positive rate is lower. As the substitution rate increases, more informative sites with a higher proportion of multi-state characters are obtained, and the alignment becomes more divergent. Furthermore, ptACR is robust even in the presence of coincident SNPs, where homoplasy is caused by the rapid evolution due to the high mutation rate instead of the structure of tree [38]. In our compatibility model, the coincident SNPs in the region would become background noise since all regional sites are shuffled to generate the null distribution of the statistic in the permutation test.

Bruen et al. [20] proposed a similar method called pairwise homoplasy index (PHI) based on pairwise incompatibility scores of the entire genome to detect the presence of recombination. They apply a permutation test on the entire alignment to obtain the Monte Carlo $p$-value for determining the significance of the observed PHI statistic. However, their method is designed only to detect whether recombination occurs anywhere in the genome. We extend the estimation from global to local scope to explore the recombination in local regions of the genome. That is, our method not only globally detects the presence of recombination events in an alignment, but locally identifies candidate breakpoints to obtain regions with distinct phylogenetic trees.

The ability to efficiently determine recombination breakpoints in bacterial genomes is especially important for analyses such as GWAS (genome-wide association studies) that attempt to statistically associate SNPs or loci with drug resistance or other phenotypes in a way informed by phylogenetic structure [39]. Uninformed of potential recombination, such studies run the risk drawing conclusions from the appearance of homoplasic sites in recombined regions (with respect to a single global phylogeny), which could be misinterpreted as evidence for positive selection at those sites.

We used ptACR to identify multiple genomic regions in a collection of $S$. aureus clinical isolates. Recombination has been previously reported for this species, but ptACR offers an efficient method to identify breakpoints where

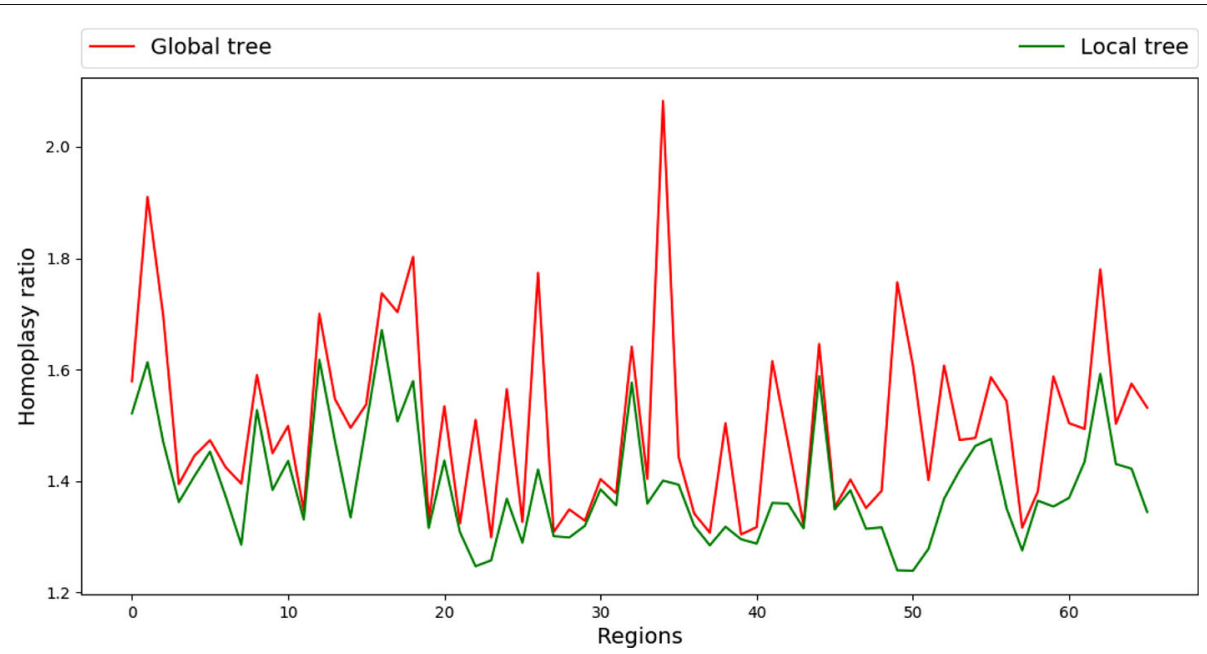

Fig. 11 Homoplasy ratio based on global and regional trees for each region of S. aureus 


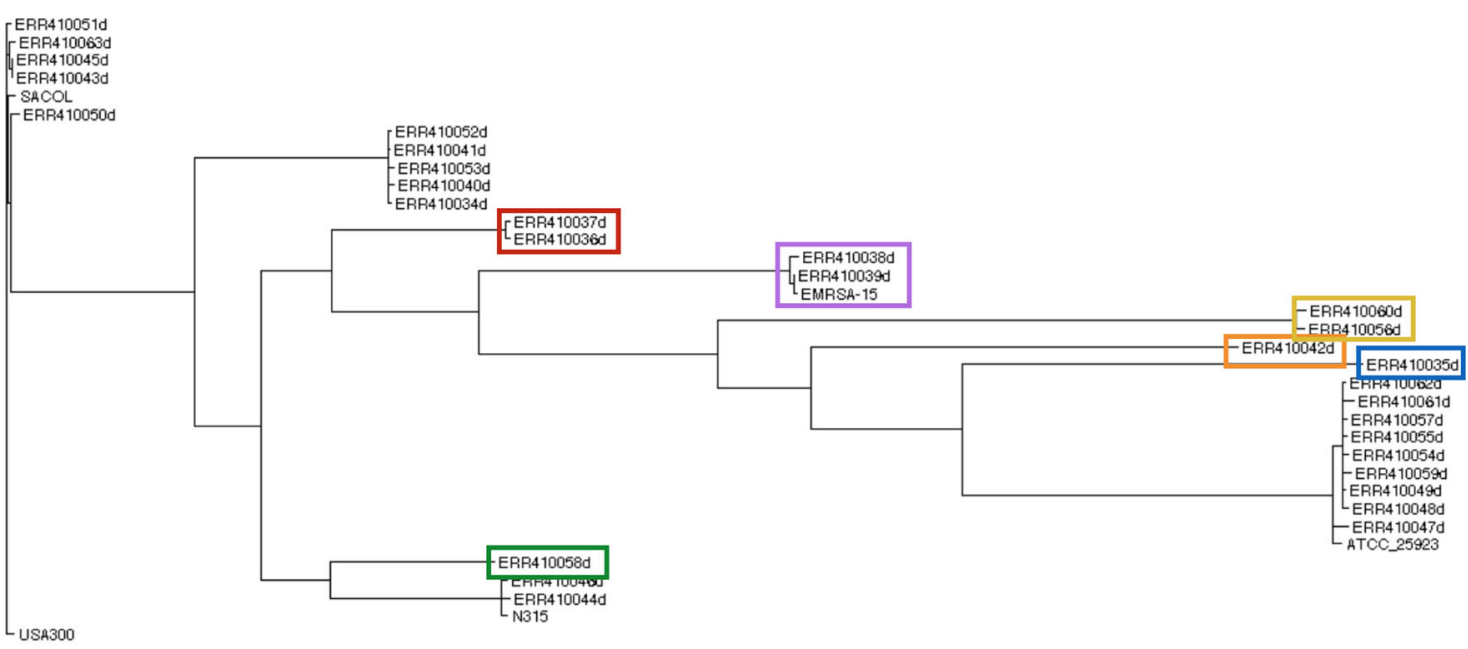

(a)

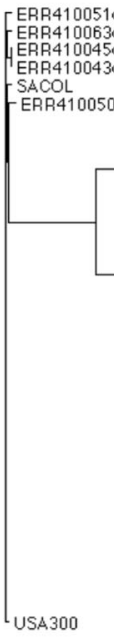

ERR410050d

ERR410053d
ERR410052d
ERR410041d
ERR410040d
ERR410034d

ERR410ก58d

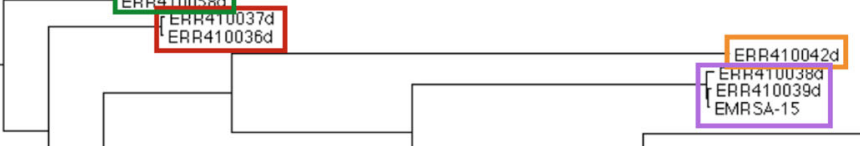

FERR410038d

ERR410060d

ERh4T00020

ERR $410057 \mathrm{~d}$

ERR410055d

ERR410059d

ERR410049d

ERR410048d

ATCC_25923

ERR410046d

(b)

ERR4100510

ERP410063

4 ERR $410045 \mathrm{~d}$

SACOL

[ERR410050d ERR410053d

ERR410052d

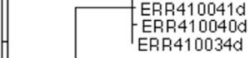

ERR410058d

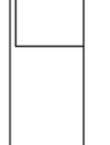

ERR410042d

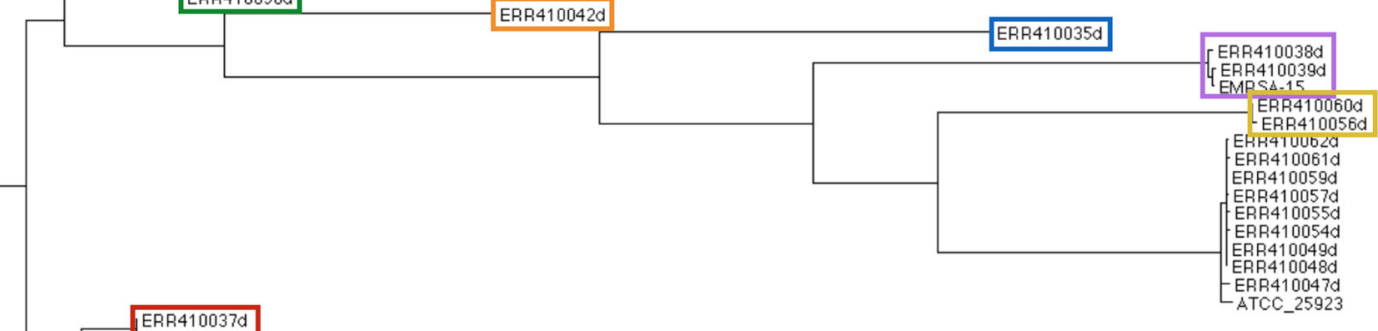

SA 300

政

ERP411046d

(c)

Fig. 12 Phylogenetic trees in the $37^{\text {th }}-39^{\text {th }}$ regions (a-c) of S. aureus 
Table 1 Information for regions of S. aureus

\begin{tabular}{|c|c|c|c|c|c|c|c|}
\hline Region & Size $(k b)^{a}$ & $\mathrm{SNPS}^{\mathrm{b}}$ & Genes $^{c}$ & Compat $^{d}$ & $E C_{\text {global }}{ }^{e}$ & $E C_{\text {local }}{ }^{f}$ & Ratio $^{9}$ \\
\hline $37^{\text {th }}$ & 228.41 & 5526 & USA300_1420-1668 & $94.59 \%$ & 1993 & 1808 & $9.28 \%$ \\
\hline $38^{\text {th }}$ & 97.74 & 4777 & USA300_1669-1747 & $93.63 \%$ & 1512 & 1400 & $7.41 \%$ \\
\hline $39^{\text {th }}$ & 36.17 & 1745 & USA300_1747-1778 & $89.93 \%$ & 914 & 577 & $36.87 \%$ \\
\hline
\end{tabular}

a region size

${ }^{b}$ number of informative sites

' genes in the region

${ }^{d}$ regional compatibility ratio

${ }^{e}$ the excess changes based on the global tree

fthe excess changes based on the local tree

9 the reduction ratio of excess changes, $1-\frac{E C_{\text {local }}}{E C_{\text {globol }}}$

the recombination events are likely to have occurred. There were 65 such breakpoints in the set of 30 isolates we analyzed. The fact that phylogenetic trees generated from SNPs in adjacent regions are distinct, coupled with the mosaic pattern of similarities to reference strains among these regions, demonstrates the validity of the ptACR method. S. aureus is a human pathogen that causes lung and skin infections. Studies have revealed that $S$. aureus contains many types of mobile genetic elements that drive recombination hotspots, including plasmids, bacteriophages, pathogenicity genomic islands and islets, transposons, insertion sequences and staphylococcal cassette chromosomes (SCC) $[12,13]$. In the collection we studied, the $28^{\text {th }}$ region contains mecA (USA30OHOU_0956) gene that is located on SCC and most commonly known as encoding methicillin resistance in S. aureus [40, 41]. Also, the $\operatorname{spA}$ gene, which is on a plasmid-associated island and contributes to staphylococcal virulence [42], is in the $37^{\text {th }}$ region.
The ptACR method has several limitations. One limitation is that, though the ability of ptACR is extended to handle an alignment consisting of multi-state characters, the pairwise compatibility for multi-state characters cannot guarantee setwise compatibility. Determining the compatibility of a pair of multi-state characters is solvable in polynomial time, however, determining the compatibility of a set of multi-state characters is NP-complete [24]. However, if two characters are incompatible, then there is no tree that can accommodate both sites at the same time. In practice, local decreases in average pairwise incompatibility is an approximate way to detect the boundaries in polynomial time.

Another limitation is that, because of the use of a sliding window, there is a practical limit on how small of a recombined region can be detected. The region has to be large enough, and the sequences diverse enough, so that region contains at least as many informative sites as the chosen window size. Finally, ptACR might be unable

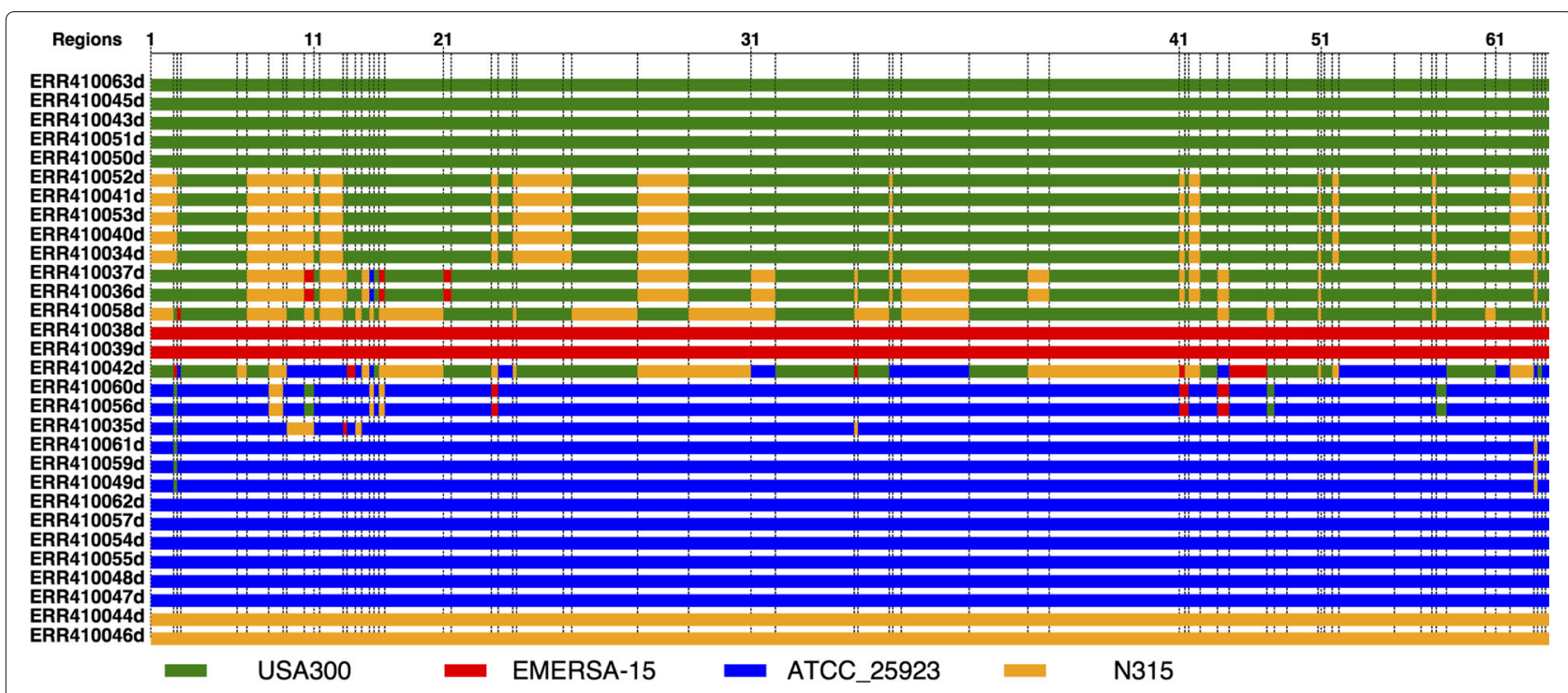

Fig. 13 Mosaic patterns plotted from the most closely related reference strains across 66 regions for 30 S. aureus strains 
to give a proper interpretation in the case of overlapping recombination regions. The phylogenetic relationships in a region overlapped by two recombination events could look different from those in the non-intersecting parts of the regions. While ptACR would likely be able to detect the boundaries of the recombined regions, it would not necessarily be able to reconstruct the exact history of the events.

\section{Conclusions}

The ptACR method is able to practically determine the compatibility of sites of binary- and multi-state characters and detect the recombination boundaries of lower average compatibility ratio with the assessment of statistical significance as candidate breakpoints. The method is sensitive, yet has a low false positive rate, supporting its ability to characterize mosaic genomes and identify the regions of distinct phylogenetic histories. With the detection of recombination events in clinical isolates of $S$. aureus, it could provide the better understanding of evolutionary relationships among bacterial isolates that is not clonal, driven by selection pressure or antibiotic resistance.

\section{Abbreviations}

ACR: Average compatibility ratio; SNP: Single nucleotide polymorphism

\section{Acknowledgements}

Not applicable.

\section{Funding}

Not applicable.

\section{Availability of data and materials}

The ptACR method, which is implemented as a Python script, is freely available at https://github.com/yplai/ptACR.

\section{Authors' contributions}

Y-PL developed the method, implemented the scripts, and performed the experiments, and Y-PL and TRI wrote the paper.

\section{Ethics approval and consent to participate}

Not applicable.

\section{Consent for publication}

Not applicable.

\section{Competing interests}

The authors declare that they have no competing interests.

\section{Publisher's Note}

Springer Nature remains neutral with regard to jurisdictional claims in published maps and institutional affiliations.

Received: 2 May 2018 Accepted: 31 October 2018

Published online: 22 November 2018

\section{References}

1. Feil EJ, Spratt BG. Recombination and the population structures of bacterial pathogens. Ann Rev Microbiol. 2001;55(1):561-90.

2. Maynard Smith J, Smith NH. Detecting recombination from gene trees. Mol Biol Evol. 1998;15(5):590-9.

3. Brandley MC, Warren DL, Leaché AD, McGuire JA. Homoplasy and clade support. Syst Biol. 2009;58(2):184-98.
4. Posada D, Crandall KA. The effect of recombination on the accuracy of phylogeny estimation. J Mol Evol. 2002;54(3):396-402.

5. Didelot $X$, Maiden MC. Impact of recombination on bacterial evolution. Trends Microbiol. 2010;18(7):315-22.

6. Krzywinska E, Krzywinski J, Schorey JS. Naturally occurring horizontal gene transfer and homologous recombination in mycobacterium. Microbiology. 2004;150(6):1707-12.

7. Marklund B, Speert D, Stokes R. Gene replacement through homologous recombination in Mycobacterium intracellulare. J Bacteriol. 1995;177(21): 6100-5.

8. Holmes EC, Urwin R, Maiden M. The influence of recombination on the population structure and evolution of the human pathogen Neisseria meningitidis. Mol Biol Evol. 1999;16(6):741-9.

9. Kong Y, Ma JH, Warren K, Tsang RS, Low DE, Jamieson FB, Alexander DC, Hao W. Homologous recombination drives both sequence diversity and gene content variation in Neisseria meningitidis. Genome Biol Evol. 2013;5(9):1611-27.

10. Didelot X, Bowden R, Street T, Golubchik T, Spencer C, McVean G, Sangal V, Anjum MF, Achtman M, Falush D, et al. Recombination and population structure in Salmonella enterica. PLoS Genet. 2011;7(7): 1002191.

11. Takuno S, Kado T, Sugino RP, Nakhleh L, Innan H. Population genomics in bacteria: a case study of staphylococcus aureus. Mol Biol Evol. 2011;29(2):797-809.

12. Everitt RG, Didelot $X$, Batty EM, Miller RR, Knox K, Young BC, Bowden R, Auton A, Votintseva A, Larner-Svensson $\mathrm{H}$, et al. Mobile elements drive recombination hotspots in the core genome of Staphylococcus aureus. Nat Commun. 2014;5:3956.

13. Driebe EM, Sahl JW, Roe C, Bowers JR, Schupp JM, Gillece JD, Kelley E, Price LB, Pearson TR, Hepp CM, et al. Using whole genome analysis to examine recombination across diverse sequence types of Staphylococcus aureus. PLoS ONE. 2015;10(7):0130955.

14. Chaguza C, Cornick JE, Everett DB. Mechanisms and impact of genetic recombination in the evolution of Streptococcus pneumoniae. Comput Struct Biotechnol J. 2015;13:241-7.

15. Kalia A, Spratt BG, Enright MC, Bessen DE. Influence of recombination and niche separation on the population genetic structure of the pathogen Streptococcus pyogenes. Infect Immun. 2002;70(4):1971-83.

16. Posada D, Crandall KA. Evaluation of methods for detecting recombination from DNA sequences: computer simulations. Proc Nat Acad Sci. 2001;98(24):13757-62.

17. Jakobsen IB, Easteal S. A program for calculating and displaying compatibility matrices as an aid in determining reticulate evolution in molecular sequences. Bioinformatics. 1996;12(4):291-5.

18. Maidak BL, Cole JR, Lilburn TG, Parker Jr CT, Saxman PR, Stredwick JM, Garrity GM, Li B, Olsen GJ, Pramanik S, et al. The RDP (ribosomal database project) continues. Nucleic Acids Res. 2000;28(1):173-4.

19. Kosakovsky Pond SL, Posada D, Gravenor MB, Woelk CH, Frost SD. GARD: a genetic algorithm for recombination detection. Bioinformatics. 2006;22(24):3096-8.

20. Bruen TC, Philippe $H$, Bryant D. A simple and robust statistical test for detecting the presence of recombination. Genetics. 2006;172(4):2665-81.

21. Croucher NJ, Page AJ, Connor TR, Delaney AJ, Keane JA, Bentley SD, Parkhill J, Harris SR. Rapid phylogenetic analysis of large samples of recombinant bacterial whole genome sequences using Gubbins. Nucleic Acids Res. 2015;43(3):15

22. Didelot $X$, Wilson DJ. Clonalframeml: efficient inference of recombination in whole bacterial genomes. PLoS Comput Biol. 2015;11(2):1004041.

23. Lai Y-P, loerger TR. A compatibility approach to identify recombination breakpoints in bacterial and viral genomes. In: Proceedings of the 8th ACM International Conference on Bioinformatics, Computational Biology, and Health Informatics, ACM-BCB '17. New York: ACM; 2017. p. 11-20.

24. Warnow TJ. Constructing phylogenetic trees efficiently using compatibility criteria. N Z J Bot. 1993:31(3):239-247.

25. LeQuesne WJ. A method of selection of characters in numerical taxonomy. Syst Zool. 1969;18(2):201-5

26. Hudson RR, Kaplan NL. Statistical properties of the number of recombination events in the history of a sample of dna sequences. Genetics. 1985;111(1):147-64.

27. Buneman P. A characterisation of rigid circuit graphs. Discret Math. 1974;9(3):205-12. 
28. Felsenstein J. Phylip-phylogeny inference package (version 3.2). Cladistics. 1989;5(163):6.

29. Sankoff D. Simultaneous solution of the rna folding, alignment and protosequence problems. SIAM J Appl Math. 1985;45(5):810-25.

30. Sjöstrand J, Arvestad L, Lagergren J, Sennblad B. Genphylodata: realistic simulation of gene family evolution. BMC Bioinforma. 2013;14(1):209.

31. Horiike T, Miyata D, Tateno Y, Minai R. Hgt-gen: a tool for generating a phylogenetic tree with horizontal gene transfer. Bioinformation. 2011;7(5):211.

32. Rambaut A, Grass NC. Seq-gen: an application for the monte carlo simulation of DNA sequence evolution along phylogenetic trees. Bioinformatics. 1997;13(3):235-8.

33. Hasegawa M, Kishino H, Yano T-a. Dating of the human-ape splitting by a molecular clock of mitochondrial DNA. J Mol Evol. 1985;22(2):160-74.

34. Kuhner MK, Felsenstein J. A simulation comparison of phylogeny algorithms under equal and unequal evolutionary rates. Mol Biol Evol. 1994;11(3):459-68.

35. Robinson DF, Foulds LR. Comparison of phylogenetic trees. Math Biosci. 1981;53(1-2):131-47.

36. Zhang K, Shasha D. Simple fast algorithms for the editing distance between trees and related problems. SIAM J Comput. 1989;18(6):1245-62

37. Huson DH, Bryant D. Application of phylogenetic networks in evolutionary studies. Mol Biol Evol. 2006;23(2):254-67.

38. Johnson PL, Hellmann I. Mutation rate distribution inferred from coincident SNPs and coincident substitutions. Genome Biol Evol. 2011;3: 842-50.

39. Chen PE, Shapiro BJ. The advent of genome-wide association studies for bacteria. Curr Opin Micrbiol. 2015;25:17-24.

40. Musser JM, Kapur V. Clonal analysis of methicillin-resistant Staphylococcus aureus strains from intercontinental sources: association of the mec gene with divergent phylogenetic lineages implies dissemination by horizontal transfer and recombination. J Clin Microbiol. 1992;30(8):2058-63.

41. Wielders C, Fluit A, Brisse S, Verhoef J, Schmitz F. mecA gene is widely disseminated in Staphylococcus aureus population. J Clin Microbiol. 2002;40(11):3970-5.

42. Murray $S$, Pascoe $B$, Méric $G$, Mageiros $L$, Yahara $K$, Hitchings MD, Friedmann Y, Wilkinson TS, Gormley FJ, Mack D, et al. Recombination-mediated host adaptation by avian Staphylococcus aureus. Genome Biol Evol. 2017;9(4):830-42.

Ready to submit your research? Choose BMC and benefit from:

- fast, convenient online submission

- thorough peer review by experienced researchers in your field

- rapid publication on acceptance

- support for research data, including large and complex data types

- gold Open Access which fosters wider collaboration and increased citations

- maximum visibility for your research: over $100 \mathrm{M}$ website views per year

At BMC, research is always in progress.

Learn more biomedcentral.com/submissions 